\title{
Influence en zone tropicale de l'amélioration des conditions d'entretien sur le rendement d'un troupeau de taurins
}

\author{
par J. PAGOT
}

Il est classique de dire que l'élevage est une des richesses de l'A.O.F., mais si l'on porte un jugement sans passion on est obligé de convenir que si l'élevage est en effet une richesse, il pourrait, mieux entretenu, avoir un rendement plus élevé.

Dans les régions pré-saharienne et sahélienne, du fait de la faible pluviosité, la culture est inexistante; l'élevage y est seule spéculation possible; s'il n'y avait pas d'animaux pour transformer les herbes spontanées de ces zones en viande et en lait, l'homme ne pourrait y vivre.

En zone guinéenne l'élevage est soit inexistant soit pratiqué suivant des techniques archaiques d'élevage extensif. Ce n'est qu'en zone soudanienne qu'on observe un but d'association entre l'agriculture et l'élevage en vue d'une utilisation rationnelle des possibilités de ces deux spéculations; le plus souvent il y a juxtaposition de l'élevage et de l'agriculture qui sont pratiqués par des ethnies différentes.

Que ce soit dans l'une ou l'autre des régions climatiques, l'animal ne reçoit comme nourriture que ce qu'il peut lui-même trouver en brousse. La transhumance corrige un peu l'alternance entre l'abondance de la saison des pluies et la disette de la saison sèche, les animaux pouvant pâturer dans les zones de décrue des fleuves qui restent vertes très avant dans la saison sèche. Malgré cela, la fin de la saison sèche est toujours catastrophique; le bétail en avril-mai est très bas d'état et la production laitière très réduite. Nous avons montré dans une précédente étude qu'en zone sahélienne au mois de mai la production laitière était à peine égale à la moitié de celle du mois de septembre.

Ayant chiffré l'influence des facteurs climatiques sur la production laitière moyenne mensuelle nous estimons que si, par une alimenta- tion suffisante, on corrigeait l'action de ces facteurs au niveau de leur valeur moyenne pendant les 5 meilleurs mois de l'année, on ferait passer la production annuelle du troupeau des vaches en lactation de 687 à 917 litres, soit une augmentation de 48 p. 100.

Ayant à déterminer au Centre de Recherches Zootechniques de l'A.O.F. les méthodes pratiques et économiques d'amélioration du bétail africain, nous avons commencé par mettre le troupeau sur lequel nous devions faire nos observations, dans des conditions aussi voisines que possible de celles de l'élevage local, de façon à avoir des termes de comparaison pour la mesure des progrès susceptibles d'être accomplis par l'application de méthodes zootechniques classiques, puis quand nous eûmes déterminé avec exactitude la valeur des productions en milieu naturel, les conditions d'entretien furent améliorées en élevant par paliers le niveau alimentaire.

La présente étude est l'exposé des résultats obtenus sur un troupeau de taurins de race N'Dama entretenu à Bamako, Soudan français, A.O.F..

\section{Les conditions climatiques}

Le climat est du type tropical avec alternance régulière $d$ 'une saison sèche et $d$ 'une saison des pluies. Les précipitations annuelles, dont la hauteur est voisine du mètre, sont réparties en 80 jours de juin à septembre; les quelques précipitations des mois de mai et octobre étant négligeables, la saison sèche, particulièrement sévère, dure pratiquement d'octobre à mai-juin.

Les températures maxima moyennes annuelles sont voisines de $34^{\circ} 5$, les températures minima moyennes de $21^{\circ} 4$ et la température moyenne annuelle se situe vers $28^{\circ}$. 
Les températures maxima sont enregistrées en avril-mai, $42^{\circ}-44^{\circ}$, et les minima en décembre, $8^{\circ}-10^{\circ}$.

Le degré hygrométrique moyen est minimum en Décembre (26 p. 100) et maximum en août-
Le troupeau d'expérience était composé exclusivement d'animaux à robe fauve unie.

\section{Mode d'entretien}

La première année, les animaux furent entre-

TABLEAU I

\begin{tabular}{|c|c|c|c|c|c|c|c|c|c|c|c|c|c|c|}
\hline & Mois & Janv. & Eévr. & Mars & Avr. & $\mathrm{Kai}$ & Juín & Juil. & Août & Sept. & oct. & Nov. & Déc. & $\begin{array}{l}\text { Chirftes } \\
\text { annuels }\end{array}$ \\
\hline Tempéra ture & $\begin{array}{l}1953 \\
1954\end{array}$ & $\begin{array}{l}28,7 \\
22,63\end{array}$ & $\begin{array}{l}28,3 \\
28\end{array}$ & $\begin{array}{l}29,4 \\
32,3\end{array}$ & $\begin{array}{l}33,3 \\
32,1\end{array}$ & $\begin{array}{l}32,2 \\
32,6\end{array}$ & $\begin{array}{c}29,3 \\
29\end{array}$ & $\begin{array}{l}27,1 \\
26,9\end{array}$ & $\begin{array}{l}26,8 \\
25,8\end{array}$ & $\begin{array}{l}27,5 \\
27,3\end{array}$ & $\begin{array}{l}28,4 \\
27,8\end{array}$ & $\begin{array}{l}28,1 \\
27,3\end{array}$ & $\begin{array}{l}24,9 \\
25,5\end{array}$ & \\
\hline $\begin{array}{l}\text { Degré } \\
\text { hygro- } \\
\text { métrique }\end{array}$ & $\begin{array}{l}1953 \\
1954\end{array}$ & $\begin{array}{l}34,6 \\
32\end{array}$ & $\begin{array}{l}30,3 \\
33,3\end{array}$ & $\begin{array}{l}32,3 \\
26,6\end{array}$ & $\begin{array}{l}35,9 \\
43,3\end{array}$ & $\begin{array}{l}51,3 \\
47,3\end{array}$ & $\begin{array}{l}62,9 \\
70,9\end{array}$ & $\begin{array}{l}79,3 \\
88,2\end{array}$ & $\begin{array}{l}80,9 \\
87,9\end{array}$ & $\begin{array}{l}73,9 \\
84,2\end{array}$ & $\begin{array}{l}71,3 \\
77,6\end{array}$ & $\begin{array}{l}52,3 \\
64,3\end{array}$ & $\begin{array}{l}43,6 \\
54,3\end{array}$ & \\
\hline $\begin{array}{l}\text { Prćcipi- } \\
\text { tations }\end{array}$ & $\begin{array}{l}1953 \\
1954\end{array}$ & & & $\begin{array}{r}33,9 \\
4,4\end{array}$ & 31,9 & $\begin{array}{l}47,8 \\
30\end{array}$ & $\begin{array}{l}172 \\
114,1\end{array}$ & $\begin{array}{l}186 \\
319,1\end{array}$ & $\begin{array}{l}346,5 \\
578,1\end{array}$ & $\begin{array}{l}243,3 \\
298,7\end{array}$ & $\begin{array}{l}59,1 \\
46,2\end{array}$ & 3,2 & & $\begin{array}{l}1088,6 \\
1425,7\end{array}$ \\
\hline
\end{tabular}

septembre ( 80 à 85 p. 100). Les minima absolus sont voisins de 10 à 15 p. 100 lorsque souffle l'harmattan en février et les maxima dépassent 97 p. 100 le matin en saison des pluies.

\section{Description du troupeau}

Les observations ont commencé en 1952 et furent faites sur un troupeau de taurins N'Dama d'environ 280 têtes dont 80 à 100 vaches. Les taurins N'Dama se rencontrent en A.O.F. dans le sud de la zone soudanienne et dans la zone guinéenne; c'est dans cette dernière que la densité est plus forte. L'effectif de cette race est estimé à 2.000 .000 de têtes dispersées dans des territoires du Soudan, de la Guinée, de la Côte d'Ivoire et de la Haute-Volta. Des exportations ont été faites à partir de la Guinée et de la Côte d'Ivoire vers l'A.E.F. et le Congo belge.

Les vaches pèsent en moyenne $230 \mathrm{~kg}$ et leur taille moyenne est de $106 \mathrm{~cm}$. Les taureaux pèsent $275 \mathrm{~kg}$ et leur taille au garrot est de $115 \mathrm{~cm}$.

La robe est généralement fauve ou pie fauve, mais dans certaines régions, du fait d'un isolèment géographique, le noir est dominant. tenus dans des conditions aussi voisines que possible de celles auxquelles sont soumis les troupeaux élevés en brousse.

Le matin, après la tétée des veaux, les vaches

TABLEAU II

\begin{tabular}{|c|c|c|c|c|c|c|}
\hline & \multicolumn{2}{|c|}{$\begin{array}{l}\text { Fin de la } \\
\text { saison sèche }\end{array}$} & \multicolumn{2}{|c|}{$\begin{array}{l}\text { Nilieu de } \\
\text { l'hivernage }\end{array}$} & \multicolumn{2}{|c|}{$\begin{array}{l}\text { Début de la } \\
\text { saison sèche }\end{array}$} \\
\hline & $\mathrm{Ma}$. & Avri? & Aodt & Sept. & Nov. & Déc. \\
\hline Humidité & 9,50 & 8,20 & 75,00 & 74,00 & 51,00 & 48,00 \\
\hline $\begin{array}{l}\text { Natières } \\
\text { Frotéiques }\end{array}$ & 1,27 & 0,70 & 1,58 & 1,47 & 1,70 & 1,80 \\
\hline $\begin{array}{l}\text { Katières } \\
\text { Eresses }\end{array}$ & 0,68 & 0,55 & 0,42 & 0,45 & 0,71 & 0,69 \\
\hline $\begin{array}{l}\text { Extractif } \\
\text { non azoté }\end{array}$ & 48,60 & 50,26 & 11,83 & 13,24 & 24,00 & 25,6 \\
\hline $\begin{array}{l}\text { Yatières } \\
\text { cellulos. }\end{array}$ & 31,90 & 31,00 & 8,23 & 9,64 & 16,50 . & 18,50 \\
\hline $\begin{array}{l}\text { Matières } \\
\text { minérales }\end{array}$ & 7,30 & 9,20 & 2,29 & 2,54 & 4,60 & 5,40 \\
\hline
\end{tabular}


partaient au pâturage où elles restaient de 8 à $17,30 \mathrm{~h}$., heure à laquelle elles rentraient à l'étable. où après la tétée des veaux elles restaient attachées jusqu'au lendemain matin.

L'abreuvement était fait à discrétion chaque jour, vers midi, au fleuve.

Nous indiquerons plus loin les améliorations apportées au régime alimentaire au cours de l'expérimentation.

Les animaux étaient pesés le matin à jeun le premier jour de chaque trimestre, le contrôle laitier des vaches fait régulièrement les 5,15 et 25 de chaque mois.

\section{Évolution de la qualité du pâturage}

Les animaux n'avaient comme nourriture que l'herbe qu'ils pouvaient consommer en brousse; raient dans la zone de décrue qui restait verte jusqu'en avril-mai, et en hivernage ils séjournaient en savane arborée.

La qualité générale des pâturages variait d'excellente en hivernage à très médiocre en saison sèche.

Le tableau II donne une idée de la variation de la qualité des fourrages récoltés sur les zones pâturées par le troupeau aux différentes époques de l'année $\left(^{*}\right)$.

\section{A. - Troupeau entretenu en élevage extensif}

INFLUENCE DES SAISONS SUR LE POIDS DES ANIMAUX.

Les comparaisons ont été faites sur 82 vaches adultes du troupeau qui étaient encore présentes à la fin des essais ; on a ainsi éliminé la variabilité

TABLEAU III - RECIME EXTENSIF

INFLUEICE DES SAISONS SUR LE POIDS DES ANIMAUX

\begin{tabular}{|c|c|c|c|c|c|c|}
\hline & $1^{\text {er }}$ Janv. & $1^{\text {er }}$ Avr. & $1^{\text {er } J u i l . ~}$ & $1^{\text {er }}$ oct. & 31 Déc. & $\begin{array}{l}\text { Gain total } \\
\text { dars l'annóe }\end{array}$ \\
\hline Poids total des vaches & 18.243 & 18.274 & 17.145 & 18.083 & 17.932 & -311 \\
\hline Veaux nés chaque trimestre & & 10 & 9 . & 20 & 17 & 50 \\
\hline Poids des veaux & & 186 & 130 & 335 & 235 & 805 \\
\hline Poids total rectifié & & 18.460 & 17.461 & 18.734 & 18.818 & +575 \\
\hline
\end{tabular}

VARIATION DE FOIDS A CHAQTE TRIIESTRE

\begin{tabular}{|c|c|c|c|c|c|}
\hline & $1^{\text {er }}$ trim. & $2^{\text {ème }}$ tri-. & $3^{\operatorname{lam} \theta}$ trim. & $4^{\grave{e} \tau=}$ trim. & Gain total \\
\hline Poids observé & +31 & -1129 & +938 & -151 & $-3 i 1$ \\
\hline Pourcentage & $+0,16$ & $-6,77$ & $+5,47$ & $-0,83$ & $-1,70$ \\
\hline Poids rectifié & +217 & -999 & +1273 & $+\varepsilon_{i}$ & +575 \\
\hline Pourcentage & $+1,19$ & $-5,41$ & $+7,29$ & $+0,44$ & $+3,15$ \\
\hline
\end{tabular}

cependant, comme sur les terrains du Centre de recherches zootechniques on trouve différents types de pâturages, reflet d'une grande diversité des sols (2), les troupeaux utilisaient au mieux les pâturages naturels : en saison sèche ils pâtu- qui aurait pu être due à l'élimination d'animaux en cours d'expérience.

(*) Analyses faites par le service de Biochimie du Laboratoire central de l'Elevage de Dakar-Hann. 
Les veaux étaient pesés à la naissance et les variations de poids des vaches au cours du trimestre ont èté corrigées en tenant compte du poids total des veaux nés dans le trimestre. Le tableau III résume l'ensemble des observations.

Le jer janvier 1953 le troupeau pesait $18.243 \mathrm{~kg}$, et le 30 déccmbrc 1953, $17.932 \mathrm{~kg}$, soit une perte de $311 \mathrm{~kg}(-1,70$ p. 100); en tenant compte du poids des veaux à la naissance le gain est de $575 \mathrm{~kg}$ ( +1 3,15 p. 100).

La courbe du graphique $n^{0} 1$ donne la variation du poids au cours de l'année; on note qu'en saison sèche, de janvier en mars, le poids est resté pratiquement stationnaire, mais que d'avril à fin juin il y a une perte très nette $: 1.129 \mathrm{~kg}$ ( $-6,17$ p. 100$)$; en tenant compte du poids des veaux nés au cours du trimestre on note une perte de $999 \mathrm{~kg}(-5,41$ p. 100).
En hivernage, de juillet à octobre, le poids du troupeau a augmenté de $938,5 \mathrm{~kg}$ (4,7 p. 100) et en tenant compte du poids des veaux, de $1.273 \mathrm{~kg}(7,29$ p. 100). D'octobre à décembre au début de la saison sèche, le.poids du troupeau a baissé de $151 \mathrm{~kg}(-0,83$ p. 100); en tenant compte du poids des veaux nés au cours du trimestre on trouve un gain de $84 \mathrm{~kg}(+0,50$ p. 100).

Tout se passe comme si au cours de l'hivernage, les animaux ne faisaient que récupérer ce quils ont perdu en saison sèche. Ainsi s'explique le manque de précocité des animaux élevés en brousse.

INFLUENCE DES SAISONS SUR LA PRODUCTION LAITIÈRE.

$\mathrm{Au}$ cours de l'année la production moyenne

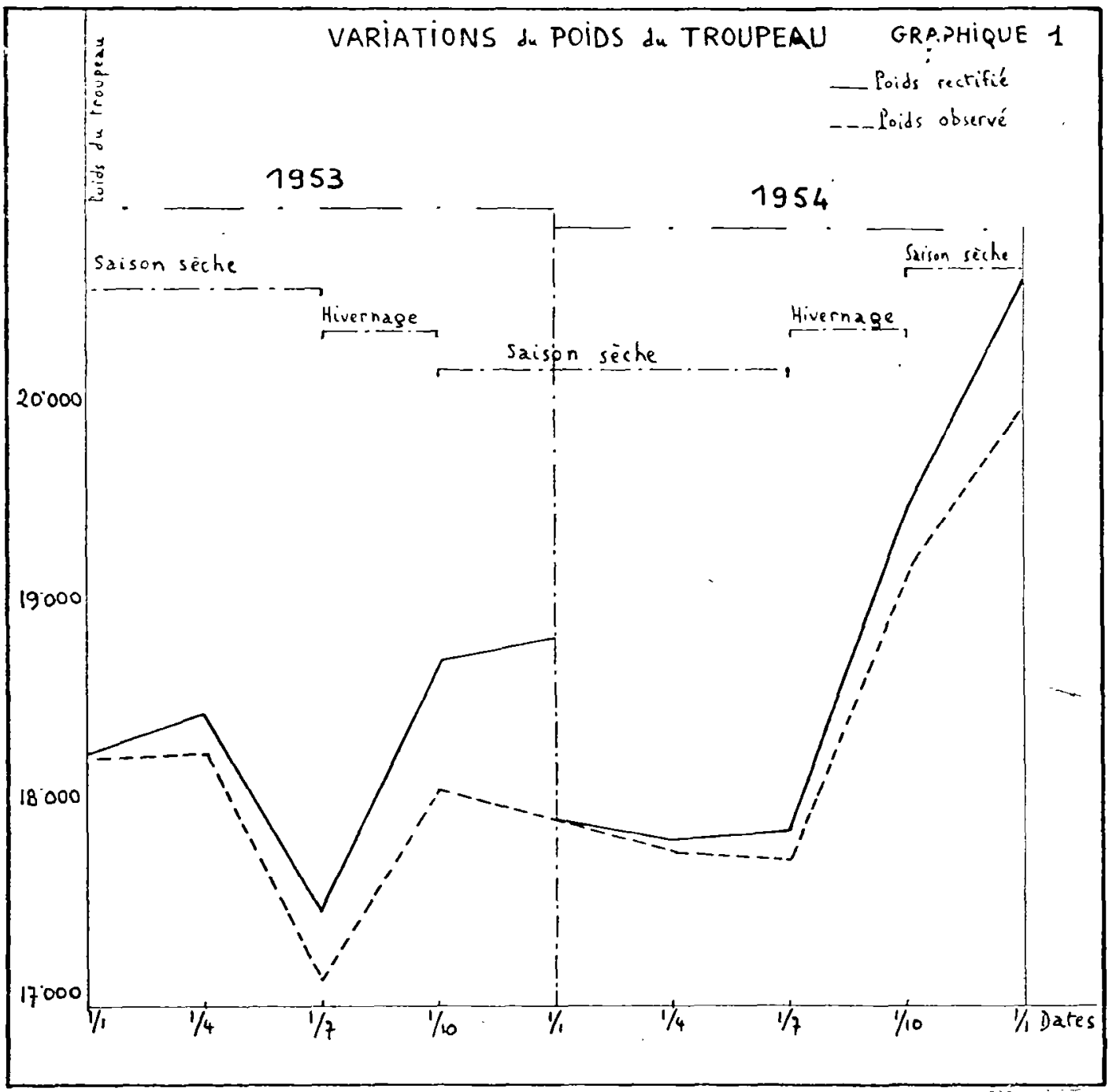


par vache du troupeau des vaches en lactation a été de $421,7 \mathrm{~kg}$ et les productions moyennes mensuelles de ce troupeau ont été par animal les suivantes :

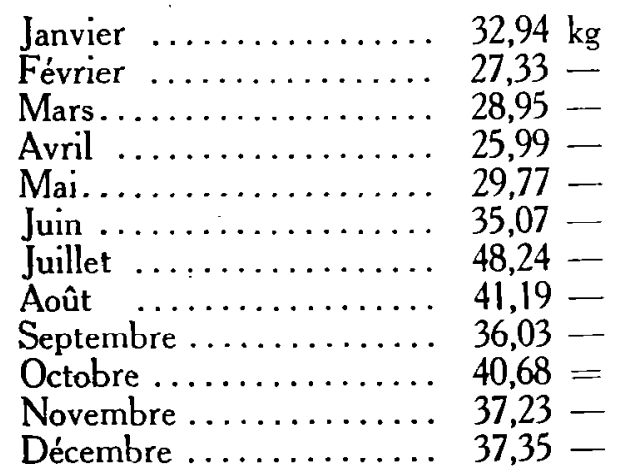

Le graphique $\mathrm{n}^{\circ} 2$ montre de façon frappante les variations des productions moyennes observées au cours de l'année ; les productions maxima ont été enregistrées en hivernage (juillet-août), les minima en avril et mai. En octobre, novembre et décembre la production s'est maintenue à un niveau acceptable du fait que le troupeau pâturait dans une zone de décrue où l'herbe était encore de bonne qualité.

\section{Conclusion.}

Des observations faites sur le troupeau élevé dans des conditions d'élevage extensif identiques à celles dans lesquelles sont entretenues les troupeaux autochtones, on peut conclure que le régime du pâturage exclusif n'a pas été suffisant pour maintenir les animaux à leur poids initial et la production laitière à un niveau normal. La perte de poids a été de 1,70 p. 100 ; le déficit de la production laitière, par rapport aux meilleurs mois de l'année, peut être évalué à près de 20 p. 100.

\section{B. - Troupeau entretenu en élevage semi-extensif}

Amélioration du milieu.

Les conditions d'élevage extensif n étant guère favorables aux animaux, nous avons en 1954 essayé de déterminer qu' elle était la ration minima quil fallait distribuer en saison sèche, pour que les animaux conservent le poids quils avaient au début de cette saison, de telle façon que le gain d'hivernage ne soit pas une simple récupération des pertes.

Nous nous sommes astreints à ne donner que des rations dont les constituants pouvaient être trouvés sur place à des prix normaux; elles étaient composées d'ensilage, de farines basses de riz, de tourteaux d'arachides, sous-produits des rizeries et huileries locales.

Si nous n'avons pas d'emblée donné aux animaux un régime riche, c'est que nous voulions que nos résultats puissent être appliqués immédiatement en brousse où il cst impossible de distribuer des suppléments pendant l'hivernage, puisque les animaux sont en transhumance ou laissés libres de vagabonder en permanence en dehors des zones de cultures.

\section{Calendrier des essais.}

Les animaux élevés dans les conditions de l'élevage extensif en 1953 ont reçu à partir du

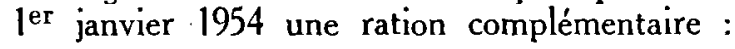

- Du 1-1-54 au 15-3-54 (75 jours) : 0,750 kg de farine basse de riz le matin, $3 \mathrm{~kg}$ d'ensilage le soir.

- Du 16-3-54 au 15-7-54 (112 jours), $0,750 \mathrm{~kg}$ du mélange suivant :

Farine basse de riz .... 77 p. 100

Tourteaux d'arachides ... 20 p. 100

Mélange minéral ...... 30 p. 100

Le mélange minéral avait lui-même la formule suivante :

Sel marin .......... 46 p. 100

Coquilles d'huitres pulvérisées ............ 46 p. 100

Phosphate tricalcique officinal ........... 8 p. 100

- Du 16-7-54 au 1-11-54 (108 jours) aucun supplément sauf pour des vaches fraîches vêlées qui ont reçu $1 \mathrm{~kg}$ du mélange précédent.

- Du 1-11-54 au 31-12-54 (61 jours) : $0,750 \mathrm{~kg}$ du mélange précédent.

Il a été distribué au troupeau au total :

Ensilage .......... $18.450 \mathrm{~kg}$

Farine basse de riz .... 13.171 -

Tourteaux d'arachides ... 2.238 -

Sel ............... 228 -

Phosphate tricalcique .... 39 -

Coquilles d'huitres ..... 228 -

Résultats.

Poids du troupeau.

En un an le poids du troupeau est passé de 17.952 à $20.010 \mathrm{~kg}$, soit un gain de $2.078 \mathrm{~kg}$ (11, 58 p. 100), ou compte tenu du poids des veaux à la naissance $2.692 \mathrm{~kg}(15,01 \mathrm{p}$. 100). 


\begin{tabular}{|c|r|r|r|r|r|r|}
\hline & $1^{\text {er Janv. }}$ & $1^{\text {er Avr. }}$ & $1^{\text {er Juil. }}$ & $1^{\text {er }}$ Oct. & 31 Déc. & $\begin{array}{c}\text { Gain total } \\
\text { dans l'arnte }\end{array}$ \\
\hline Poids total des vaches & 17.932 & 17.760 & 17.716 & 19.209 & 20.010 & +2.073 \\
Veaux nés chaque trimestre & & 4 & 8 & 9 & 20 & 41 \\
Poíds des veaux & & 52 & 113 & 136 & 313 & 614 \\
Poids total rcctifié & & 17.812 & 17.881 & 19.510 & 20.624 & +2.692 \\
\hline
\end{tabular}

VARIATION DE PUILS AU CCURS DU TRIVZOTRE

\begin{tabular}{|c|c|c|c|c|c|}
\hline 1 & $1^{e r}$ trim. & $2^{\text {ème }}$ trim. & $3^{\text {ème }}$ trim. & $4^{\text {ème }}$ trim. & $\begin{array}{l}\text { Total de } \\
\text { I'année }\end{array}$ \\
\hline Poids observé & -172 & -44 & +1493 & + 801 & +2078 \\
\hline Pourcertago & $-0,95$ & $-0,24$ & $+8,42$ & $+4,16$ & $+11,58$ \\
\hline Poids rectifié & -120 & +69 & +1629 & +1114 & 2592 \\
\hline Pourcentage & & $+0,38$ & & & 15,01 \\
\hline
\end{tabular}

La courbe du graphique I donne l'allure des variations de poids enregistrées au cours de l'année.

$\mathrm{Au}$ cours du premier trimestre lorsqu'on n'a distribué que de la farine de riz et de l'ensilage le poids du troupeau a, en tenant compte du poids de veaux, légèrement fléchi $(-120 \mathrm{~kg}$, 0,67 p. 100).

$\mathrm{Au}$ cours du second trimestre le poids est resté stationnaire $(+69 \mathrm{~kg}, 0,38 \mathrm{p} .100)$ alors que l'année précédente l'amaigrissement se chiffrait compte tenu du poids des veaux à 999 kg (5,41 p. 100).

Les animaux abordant l'hivernage en bon état, le gain fut au cours du troisième trimestre de $1.629 \mathrm{~kg}(9,11$ p. 100) contre $1.273 \mathrm{~kg}(7,29$ p. 100) en 1953.

Au cours du dernier trimestre le gain fut de $1.114 \mathrm{~kg}(5.71$ p. 100$)$ contre $84 \mathrm{~kg}(0,44$ p. 100) en 1953 .
Ainsi la distribution dune ration relativement faible a permis de maintenir le poids du troupeau pendant la saison sèche pratiquement constant. et surtout de gagner un poids notable au cours de l'hivernage et au début de la saison sèche.

\section{Production laitière.}

La production moyenne annuelle par vache du troupeau des vaches en lactation a été de $491,4 \mathrm{~kg}$ contre 421,7 en 1953 soit un gain de $69,7 \mathrm{~kg}$ (16,5 p. 100).

Les productions moyennes mensuelles de ce troupeau ont été les suivantes:

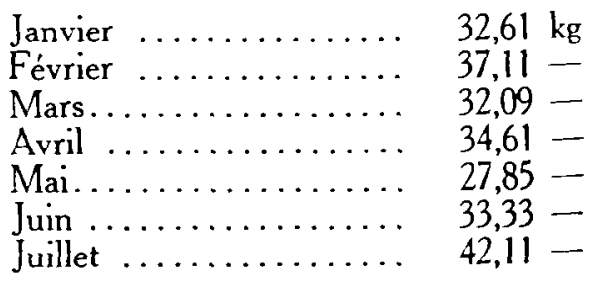




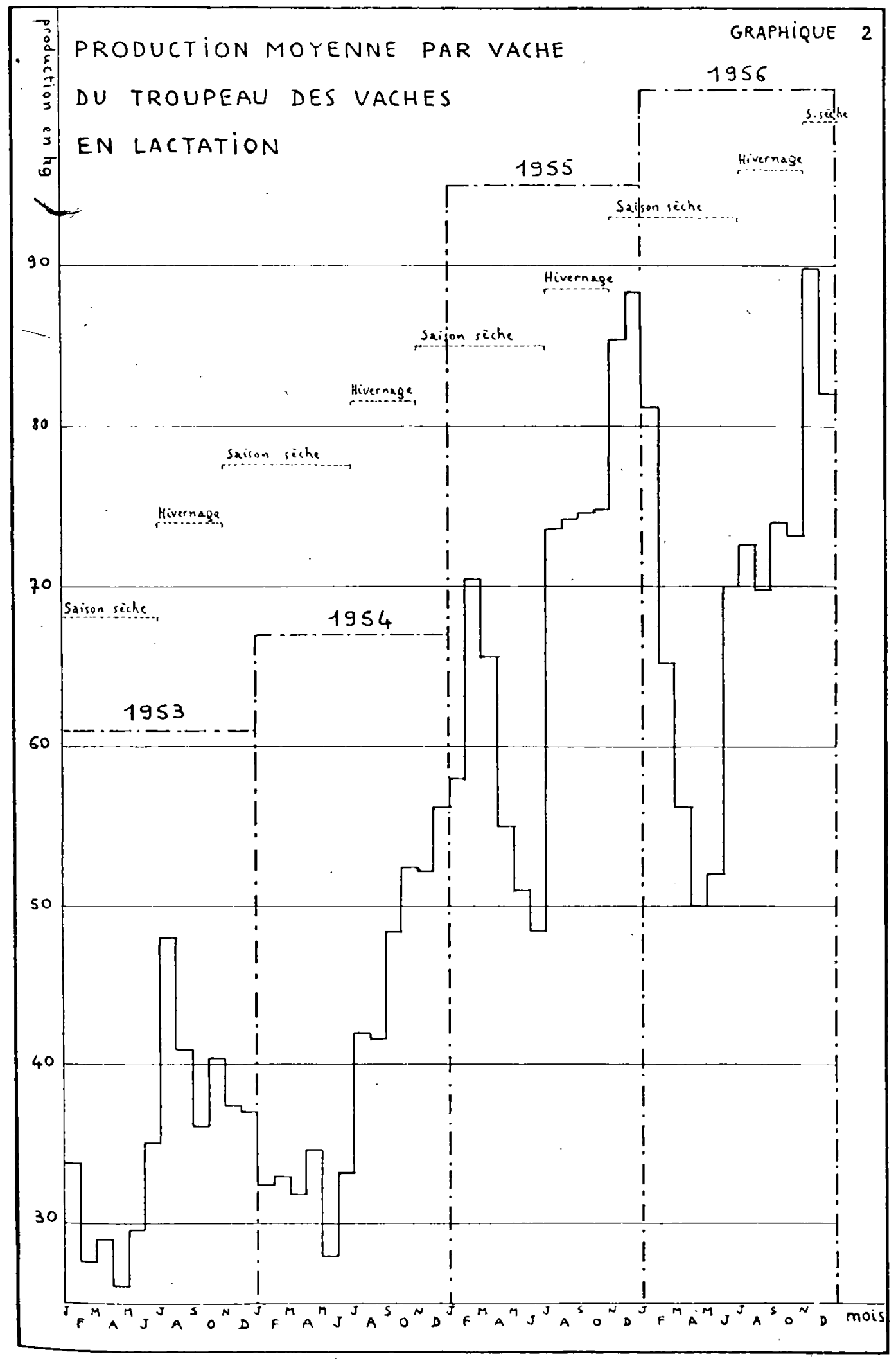


Août

Septembre

$41,87-$

$48,48-$

Octobre ............. $52,36-$

Novembre ........... 52,26 -

Décembre .......... 56,32

Total $\ldots \ldots \ldots$... 491,39 -

$\mathrm{Si}$ on compare les courbes des productions
Le prix de revient de l'ensilage comprend tous les postes dépenses (façon culturales, récoltes, conservateurs, entretien et amortissement du matériel).

Le surplus de lait a été estimé en fonction du nombre de vaches en lactation qui a varié suivant les mois entre 21 et 50 . Les lactations

TABLEAU V

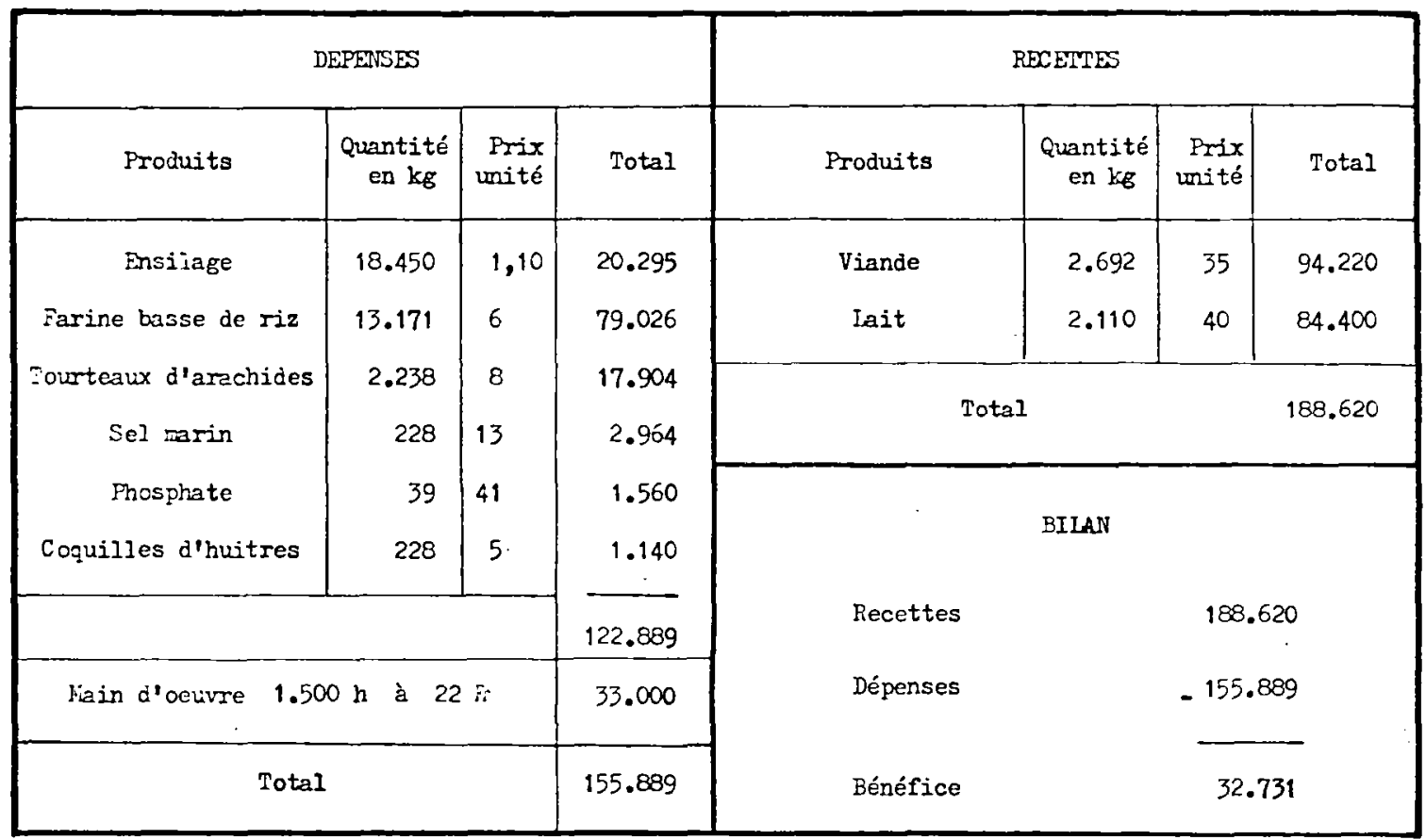

moyennes mensuelles du troupeau des vaches en lactation pour 1953 et 1954, on note que pendant les mois de saison sèche d'avril-mai, la baisse de production s'est produite au cours des deux années mais que les productions minima en 1954 étaient supérieures à celles de 1953.

En 1954, la production n'a cessé de croître jusqu'à la fin de l'année alors qu'en 1953 on notait une baisse notable au cours du dernier trimestre.

\section{Rentabilité de lopération.}

Nous avons essayé de dresser le bilan de l'opération en tenant compte des prix de revient de l'ensilage, des aliments concentrés et des prix de la viande et du lait pratiqués à Bamako, celui du lait étant volontairement sous-estimé.

On a compté qu'il fallait un homme pendant deux heures matin et soir pour distribuer les aliments. étaient arrêtées volontairement à la fin du $7^{\mathrm{e}}$ mois.

Ainsi, grâce à un appoint relativement faible d'aliments disponibles sur place, le troupeau a été maintenu pendant la saison sèche, le gain de poids au cours de l'année a été substantiel et la production laitière a été augmentée dans de notables proportions. Le bilan de l'opération est positif, il se solde par un gain net de $32.731 \mathrm{fr}$, main d'cuvre payée.

$\mathrm{Si}$ on dresse le bilan en unités fourragères on trouve que les aliments distribués ont une valeur bien plus élevée que la valeur théorique de ceux nécessaires pour obtenir les productions supplémentaires récoltées; ceci s'explique par la qualité des pâturages moindre en 1954 qu'en 1953.

L'herbe consommée au début de l'année 1953 correspondait à la pousse de l'hivernage 1952 au cours duquel il était tombé $1.264 \mathrm{~mm}$ d'eau, 
alors qu'en 1954 les animaux ont consommé l'herbe de l'hivernage 1953 au cours duquel il n'était tombé que $1.088 \mathrm{~mm}$; les suppléments distribués ont donc servi à pallier la moindre qualité des fuurrages et à augmenter la production du troupeau.

Nous donnerons dans un prochain article les résultats d'essais d'alimentation intensive faits sur des lots d'animaux N'dama.

\section{Conclusion.}

En nutilisant que les produits du pays on peut augmenter dans de notables proportions le rendement des troupeaux et valoriser ainsi des sous-produits de cultures vivrières ou industrielles locales qui sont soit exportés (tourteaux) soit utilisés comme engrais (farines basses de riz).

\section{VÉRIFICATION DES RÉSULTATS.}

En 1955-1956, les observations n'ont pas pu être poursuivies sur la totalité des 82 vaches qui avaient servi en 1953-1954. En effet certaines étaient devenues trop âgées; d'autres s'étaient révélées être de très mauvaises laitières. Les observations furent donc faites sur 41 vaches adultes.

\section{Calendrier des essais.}

En 1955-1956 le mélange concentré distribué avait la composition suivante :

Tourteaux d'arachide ......... 48

Farine basse de riz ........... 50

Mélange minéral ............ 2

La valeur alimentaire était de 1,07 U.F. par kg; les quantités distribuées en 1955-1956 furent les suivantes :
I-I au 31-3 (90 jours) :

\begin{tabular}{lll} 
Ensilage $\ldots \ldots \ldots \ldots \ldots$ & 3 \\
Mélange concentré & $\mathrm{kg}$ \\
\hline
\end{tabular}

1-4 au 14-9 (168 jours) :

Mélange concentré ....

15-9 au 31-12 (107 jours) :

Mélange concentré

$0,500-$

Poids des animaux.

Les 41 vaches qui formaient le lot d'observation étaient à la fin de l'année 1954, en excellent état d'entretien et pesaient en moyenne $253 \mathrm{~kg}$. Nous rappelons pour mémoire que les vaches pesaient au début de l'année 1953:225 kg, et de l'année $1954: 221 \mathrm{~kg}$. L'excellent état d'entretien fut maintenu tout au long de l'expérience.
Le gain fut encore en 1955 de 3,3 p. 100 et en 1956 de 2,8 p. 100.

\section{Production laitière.}

Pour la production laitière il n'est pas possible de porter la totalité des progrès au compte de l'amélioration du milieu puisqu'il y eut une certaine sélection parmi le troupeau initial.

La production laitière moyenne annuelle par vache du troupeau des vaches en lactation a atteint en 1955 et 1956 respectivement $830,54 \mathrm{~kg}$ et $842,19 \mathrm{~kg}$, contre 491,39 en 1954 , et 421,67 en 1953, soit pratiquement le double.

Compte tenu de la remarque faite ci-dessus, relative à l'élimination des mauvaises laitières, on notera le progrès accompli, quand on saura que chaque mois il y avait de 25 à 40 vaches en lactation et que 15 vaches réformées avaient été remplacées par des primipares.

Les productions mensuelles moyennes par animal du troupeau des vaches en lactation ont été les suivantes :

\section{5}

1956

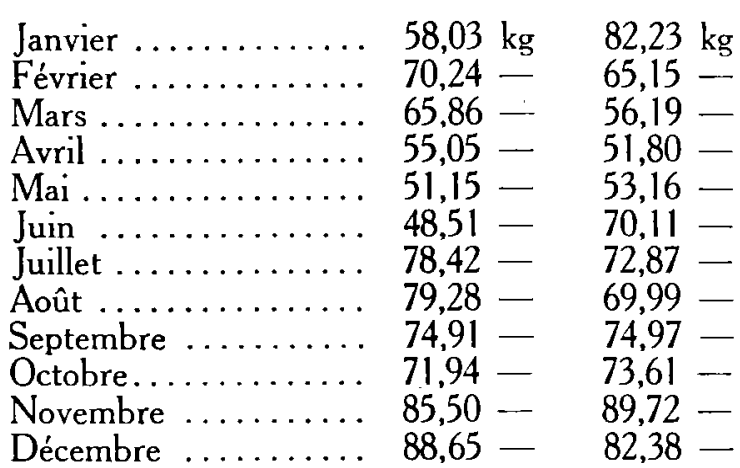

$\mathrm{Si}$ on se reporte au graphique $\mathrm{n}^{0} 1$ on y observe toujours la dépression de la fin de la saison sèche, mais on peut remarquer que les minima de la saison sèche de 1955 sont voisins des maxima de la saison des pluies de 1954, et que la production d'avril 1955 est supérieure à celle de septembre 1954. La courbe générale reste la même, mais l'accroissement dû̀ à l'hivernage atteint presque, dès le premier mois, son maximum.

\section{ConClusion.}

Ainsi les observations faites en 1955-1956 ont confirmé celles de 1954.

En distribuant aux animaux allant dans la journée au pâturage, une ration de complément 
dont la valeur est voisine de l'unité fourragère, on peut éviter aux animaux l'amaigrissement qui survient à la fin de la saison sèche et on augmente dans de notables proportions, la production laitière.

En améliorant les conditions d'entretien, en 1954, nous avons obtenu un gain de production de lait de 16,52 p. 100 et la mise en état du troupeau. Quand cette mise en état fut obtenue, l'amélioration des conditions d'entretien, jointe à la sélection, a permis d'augmenter la production laitière de $90 \mathrm{p} .100$.

Ainsi il est donc prouvé qu'avec des moyens très simples il est possible d'accroittre la productivité du troupeau local en n'utilisant que des produits disponibles sur place. En effet les tourteaux d'arachide et de palmiste sont abondants en A.O.F. puisqu'ils sont exportés et les farines basses de riz sont généralement utilisées comme engrais pour les rizières.

Centre de recherches zootectniques, Sotuba Bamako.

\section{BIBLIOGRAPHIE}

1. AdARi - Inventaire botanique des pâturages du centre de recherches zootechniques de I'A.O.F. A paraitre.

2. Charreau et Dommergues. - Etude des sols du centre de recherches zootechniques de I'A.O.F. A paraitre.

3. Derbal, Pagot et Lahore. - Résumé synthétique des recherches faites sur les pâturages tropicaux de la région soudanienne au centre de recherches zootechniques de l'A.O.F. A paraitre.

4. Pagot. - La race de IAzawack. Bull. Serv. zoot. épiz. A.O.F., 1943, 6, 155-63.

5. Pagot. - Production laitière en zone tropicale; faits d'expérience en A.O.F. Rev. Elev. Méd. vét. Pays trop., 1951-52, 5, 173-90.

\section{SUMMARY}

\section{The Influence on the Productivity of a Herd of Humpless Cattle in a Tropical Zone by Improvement of their Maintenance Conditions}

The author experimented on a herd of the N'Dama breed being reared in Bamako in the French Sudan. He describes briefly the local climatic conditions, details of the herd, and its management, and describes the changes in the quality of the forage available at various seasons of the year. This herd placed under the same conditions as local breeds shows during the period of the dry season a loss of 17 p. 100 of liveweight and a 20 p. 100 reduction in milk production, as compared to the standards during the better months of the year. The author has made up a complementary feeding ration utilising only local products (rice flour, groundnut cake, and minerals) given to animals yoing out grazing. With this ration he has prevented this seasonal loss of condition and practically doubled the milk production. The cost of this supplementary ration is sufficiently low to be economic.

\section{RESUMEN}

\section{Influencia en la zona tropical, del mejoramiento de las condiciones] de mantenimiento sobre el rendimiento de un rebaño de taurins}

$\mathrm{El}$ autor ha experimentado con un rebaño de taurins N'Dama mantenido en Bamako (Sudan, A.O.F.). Rapidamente cita las condiciones climáticas, describe el rebaño y su sistema de mantenimiento y examina la evolución de la calidad del forrage durante el año. Estudiando el rebaño puesto en las mismas condiciones que los rebaños autóctonos, constata que con relación a los mejores meses del año, durante los meses de la estacion seca hay una pérdida de peso del $17 \mathrm{p} .100 \mathrm{y}$ un Ceficit de la produccion lechera del 20 p. 100.

El autor ha calculado una ración suplementaria utilizando solamente productos locales (harina de arroz de baja calidad, tortas de cacahuete, sales minerales) que se da a los animales que van a pastoreo (alrededor de una unidad forregera por animal). Asi se evita el enflaquecimiento periodico y se dobla praticamente la produccion de leche ; el precio de costo de éste suplemento en bastante bajo y el mejora miento obtenido produce zanancias. 